\title{
A walk along the steep neutron resonance data evaluation path
}

\author{
Olivier Bouland ${ }^{\mathrm{a}}$, and the Cadarache Nuclear Data Group ${ }^{\mathrm{b}}$ \\ Physics Studies Laboratory, CEA Cadarache, Bd 230, DEN/CAD/DER/SPRC/LEPh, 13108 Saint-Paul-lez-Durance, France
}

\begin{abstract}
This paper goes through some major steps of a "resonance evaluator" work and in particular on actual tricky questions: the nature of the experimental data base, the life without $R$-matrix approximations, the external level gambling, the influence of level structures in observables other than cross sections, the puzzling unresolved resonance range and the disused average sub-threshold fission cross sections.
\end{abstract}

\section{Introduction}

The aim of this paper is to walk on the steps of a "resonance evaluator" but also to focus on the present questions relative to his job. At the commencement, there are both differential and integral experimental results. In the past these two sorts of data were opposed. The second section will demonstrate the complementarity of these data and will give an example of their use in a neutron re-evaluation work. Right-after the selection of the experimental data base comes the $R$-matrix data analysis and the collection of the associated $R$-matrix parameters. $R$-matrix theory was in the past rudely approximated on the form of the Single-Level Breit-Wigner (SLBW) formulation and this is not rare to meet resolved resonance evaluations based on this simple prescription. Nowadays there is no more reasons to use this formalism or its Multi-Level version (MLBW); the less restrictive approximation proposed by Reich and Moore (RM) must always be favoured. The third section will debate on the impact of this RM approximation relatively to a genuine $R$-matrix calculation. The discrepancy observed on the capture cross section (XS) value is somehow compensated by the hazardous modelization of the external levels (EL). On this latter point, various ad hoc recipes exist and this paper will show in section 4 that there are nearly as many methods as existing resonance evaluators. Section 5 will present the progresses made on the evaluation of the isomeric ratio and the effect of level structure on it. Section 6 is devoted to the twilight zone, the so-called unresolved resonance region (URR) at midway between the Resolved Resonance Range (RRR) and the continuum. The last item debated in this paper concerns the disused fission XS of the non fissile heavy nuclei in the URR. Although of small neutronic impact in LWR reactor, this latter must not be neglected at least because more rigorous methods exist for evaluating the so-called subthreshold fission XS. Two philosophies must be distinguished: the analytical approach and the Monte Carlo simulation. This paper will analyse the possibilities offered by the two methods.

\footnotetext{
${ }^{a}$ Presenting author, e-mail: olivier.bouland@cea.fr

${ }^{b}$ Detailed member list in "Acknowledgements section"
}

\section{No future without integral and differential data}

Although numerous resonant XS data analyses were performed from experimental data bases including only differential measurements, the ideal approach must be based on all sources of information available. Such an approach was recently used for a revision [1] of the JEFF3.1 ${ }^{241} \mathrm{Am}$ resolved resonant parameters (RRP). Several analyses of reactor experiments using the neutronic code APOLLO2 and its associated JEFF3.0 application library were at the origin of this latter revision. They revealed a possible under-estimation of $\sigma_{\gamma}\left({ }^{241} \mathrm{Am}\right)$ in the thermal and epithermal energy ranges which was not foreseen using uniquely the microscopic experimental data base of JEF2.2. Indeed the old JEF2.2 RRP set (kept identical for JEFF3.0) was only based on microscopic measurements, which were showing a reasonable agreement on the thermal total XS value as can be seen in table 1 .

An exhaustive synthesis [2] was therefore made between the experimental data (microscopic differential and microscopic integral data), the major evaluated data files and the nuclear data trends derived from the validation of the JEFF3.0 library. The comparisons made have underlined large discrepancies on the thermal capture XS value between the differential measurements and the juxtaposed set of integral data. It happens now that differential results are in general significantly smaller than integral data (partially displayed in table 2) which show also a larger range of variation $\left[(625 \pm 35) \mathrm{b} \leq \sigma_{\gamma}^{0} \leq(853 \pm\right.$ 52)b]. The reasons for such a disagreement have to be found in the fact that ${ }^{241} \mathrm{Am}$ exhibits two strong resonances at $0.31 \mathrm{eV}$ and $0.58 \mathrm{eV}$. These resonances contribute strongly to the measured yield making integral measurements largely dependent on their actual reactor spectrum which is not necessarily well known. Subsequently, thermal capture XS values obtained

Table 1. ${ }^{241}$ Am differential results available at thermal energy.

\begin{tabular}{llll}
\hline Measurement type & transmission & transmission & absorption \\
Year & 1976 & 1955 & 1976 \\
First author & Kabelin & Adamchuck & Weston \\
EXFOR \# & 40305 & 40063 & 10767 \\
$\sigma_{\gamma}^{0}[b]$ & 623.5 & 586 & $579 \pm 6$ \\
\hline
\end{tabular}


Table 2. Example of ${ }^{241} \mathrm{Am}$ integral results available.

\begin{tabular}{llllll}
\hline Year & 2006 & 2001 & 1997 & 1976 & 1969 \\
Author & Bringer & Maidana & Shinohara & Gavrilov & Dovbenko \\
EXFOR & 22941 & 31518 & 22346 & 40467 & \\
Ref. & & & & \multicolumn{2}{c}{ LA-TR-71-74 } \\
$\tilde{\sigma}_{\gamma}[b]$ & $714 \pm 23$ & $678 \pm 10$ & $797 \pm 31$ & $853 \pm 52$ & $647 \pm 104$ \\
\hline
\end{tabular}

from integral experiments are essentially effective capture cross sections (noted $\tilde{\sigma}_{\gamma}$, not $\sigma_{\gamma}^{0}$ ). The measured effective XS can be much larger than the true thermal value $\left(\sigma_{\gamma}^{0}\right)$ and must be corrected of the fraction of the epithermal to the thermal fluxes, of the Westcott factor and of the self-shielding factors in the epithermal and thermal ranges. It explains why very large capture XS values were sometimes encountered in the past (e.g., by Gavrilov et al.; table 2). Nevertheless, among the old integral experiments, the measurement by Dovbenko et al. was especially designed for a direct retrieval of the 2200-m/sec capture XS. Their measurement, performed in a reactor thermal column, yielded a value of $(647 \pm 104) \mathrm{b}$ fully compatible with the differential data base.

Recent integral experiment results have demonstrated that the experimentalists are now aware of the importance of a correct determination of the shelf-shielded neutron flux. Among the three most recent integral values, two of them, respectively by Berger et al. and Maidana et al. (table 2), tend to get closer to the differential results. The authors were very careful when retrieving the true thermal XS. The third one, by Shinohara et al. (see table 2), still shows a very large measured $\mathrm{XS}$ value. This trend is also clear on their measured resonance integral value $\left(I_{R}^{\gamma}=(1897 \pm 93)\right.$ b $)$ relatively to the JEFF3.1 value $\left(I_{R}^{\gamma}=1525 \mathrm{~b}\right)$ [3]. Such large values essentially show up in integral measurements based on $\mathrm{Cd}$ ratio technique which requires a scrupulous reduction data procedure. The result depends also strongly of the effective cut-off energy (function of the Cd thickness) which none sharp value falls into the range of the two lowest ${ }^{241} \mathrm{Am}$ resonances.

Finally, the JEFF31 revision of the ${ }^{241}$ Am RRP set was performed on a $\sigma_{\gamma}^{0}$ basis of $647 \mathrm{~b}$, which is derived from the careful study of all information sources. This value appears to be $6 \%$ higher than the JEF2.2 reference value chosen by F. Fröhner [4] from only differential results.

\section{Are RRR $\boldsymbol{R}$-matrix approximations still needed?}

Since Wigner and Einsenbud [5], resonances can be exactly described by $R$-matrix theory. But this rigorous analytical theory has been always approximated for practical reasons. Although the MLBW-endf prescription might be applied to compound nuclei with narrow resonances widely spaced as in the ${ }^{238} \mathrm{U}+\mathrm{n}$ and ${ }^{232} \mathrm{Th}+\mathrm{n}$ systems, the safest procedure is to stick to the least restricted hypothesis (RM). Unfortunately, this is not rare to meet RRP evaluations involving the lowest level of prescription (SLBW-endf) although this latter is notorious for the occurrence of non-physical negative total and elastic cross sections. A recent overview of the JEFF3.1 general-purpose neutron data library [3] has still inventoried 13 nuclei $\left({ }^{89} \mathrm{Y},{ }^{93} \mathrm{Nb},{ }^{102} \mathrm{Ru},{ }^{111,113} \mathrm{Cd},{ }^{165} \mathrm{Ho},{ }^{n a t} \mathrm{Tl},{ }^{229} \mathrm{Th}\right.$, ${ }^{243,244,247} \mathrm{Cm},{ }^{247} \mathrm{Bk}$ and ${ }^{250} \mathrm{Cf}$ ) based on SLBW-endf.
Nowadays, several programs around the world (e.g., SAMMY [6], EDA [7], RAC [8]) have the capability to handle full $R$-matrix calculations. This gives the opportunity to answer to the recurrent question "what is the error due to the RM approximation?" and also to the subsidiary question "knowing this error, is it worthwhile to do a $R$-matrix calculation?".

Some attempts were made to answer to these questions by R.E. Chrien during a seminar in 1978 on the ${ }^{238} \mathrm{U}$ target nucleus and more recently by M. Moxon on ${ }^{238} \mathrm{U}$ using REFIT [9] and fictitious channel widths values. This question was also commented by F. Fröhner [10] who expected a small error due the RM approximation. In 2005, O. Bouland has made a reliable comparison [11] using the same code (SAMMY) and the same algorithm for the RM and $R$-matrix calculations in order to avoid numerical disagreements. This latter calculations were applied to real cases using ${ }^{23} \mathrm{Na},{ }^{19} \mathrm{~F}$ and ${ }^{238} \mathrm{U}$ capture gamma-rays measurements.

\subsection{Recall on the Reich-Moore approximation}

Reich and Moore have proposed to eliminate a particular type of channel (e.g., the capture channels) by assuming no crossterms from level to level in the inverse level matrix $A^{-1}$ for this particular type of channel; assumption which allows the aggregate of the diagonal contributions of these eliminated channels (i.e., for capture in the total capture width). This special treatment is well-suited for capture because the decay amplitudes $\gamma_{\lambda \gamma}$ of the many photon channels for a given level $\lambda$ have practically random signs but comparable amplitudes and then the many photon-photon cross-terms tend to cancel in the off-diagonal elements of the level matrix $A^{-1}$. But for a light nucleus which number of $\gamma$-transitions is limited, this hypothesis could fail.

\subsection{R-matrix impact on a light target nuclide}

The author of ref. [11] has shown two light target nuclide examples. Figure 1 presents the differences observed on the ${ }^{19} \mathrm{~F}$ capture XS between R-M and $R$-matrix using 17 identified partial radiative widths for the description of 3 resonances. The differences show up in the wings of the resonances of same $J^{\pi}\left(1^{-}\right)$which interfere. The magnitude of the net "interference" effect is evaluated using the total capture resonance integral $\left(I_{R}^{\gamma}\right)$ weighted by a $1 / E$ flux. This latter quantity, centred around the $49 \mathrm{keV}$ resonance $\left(37<E_{n}<\right.$ $65 \mathrm{keV})$, shows a small but significant decrease $(-1.3 \%)$. A more detailed examination of this result shows a cancellation between the left $\left(E<E_{R}\right)$ and the right sides $\left(E>E_{R}\right)$ of the resonance (respectively $+10.03 \%$ and $-12.44 \%$ ). This safe numerical compensation could be less comfortable when a broadening with an asymmetric function is required for the XS calculation. This $-1.3 \%$ "interference" effect is in fact the maximum expected "interference" effect since the partial capture amplitudes are all assigned positive in this calculation. The partial capture amplitude signs, although not given by the measurement, must be distributed randomly according theory. Therefore the genuine absolute 'interference' effect is plausibly smaller than $1.3 \%$. 


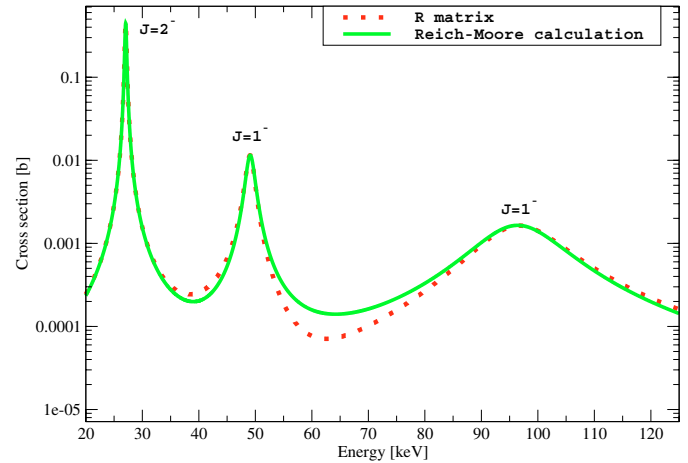

Fig. 1. ${ }^{19} \mathrm{~F}$ asymmetrical resonance shape induced by a $R$-matrix treatment (dotted curve) of $\sigma_{\gamma}$.

\section{3 $R$-matrix consequence on a heavy target nuclide}

As explained before, it is assumed for a heavy target nuclide that the many photon-photon cross-terms cancel in the offdiagonal elements of the level matrix $A^{-1}$ and subsequently motivate the use of the R-M approximation. Is this assumption still verified in the case of a heavy target nucleus exhibiting a few favoured radiative channels as for instance ${ }^{238} \mathrm{U}$ ? To clarify this point, some $R$-matrix calculations [11] were performed on this latter nucleus. They are based on the old ${ }^{238} \mathrm{U}$ capture gamma rays measurements by Price et al. [11] who identified $23 \gamma$-ray lines between $3200 \mathrm{keV}$ and $4802 \mathrm{keV}$ for the six energy lowest resonances. These resonances are responsible for $81 \%$ of the capture XS at the thermal energy $(0.0253 \mathrm{eV})$ but the 23 partial capture channels explicitly treated in this $R$-matrix calculation represent only $26 \%$ of the total capture width $\left(\Gamma_{\gamma_{t o t}}\right)$ which means that the $R$-matrix calculation is here a pseudo $R$-matrix calculation with a residual aggregated capture width equal to $74 \%$ of $\Gamma_{\gamma_{t o t}}$. These ${ }^{238} \mathrm{U}$ capture XS calculations were based on a parameter file reduced to six resonances with no bound levels. The ${ }^{238} \mathrm{U}$ parameters were extracted from JEFF3.1 except the capture widths borrowed from Price et al. Two pseudo $R$-matrix calculations were performed. The first one, similarly to ${ }^{19} \mathrm{~F}$ maximised the $R$-matrix effect since all the partial capture amplitudes were chosen of same signs in the calculation. At the opposite the second one, whose partial capture amplitudes were alternatively assigned plus or minus, gives a more realistic effect. Clearly, the major impact shows up below the first resonance. The most realistic calculation (see fig. 2, solid curve) gives $\sigma_{\gamma_{t g}}^{0}$ equal to $1.88 \mathrm{~b}$ (13\% lower than the reference RM value; long-dashed curve on fig. 2). But the residual capture, representing the eliminated partial capture channels contribution (74\% of $\Gamma_{\gamma_{t o t}}$ ), still smooth out the final result which could show a larger RM- $R$-matrix difference.

\section{4 $R$-matrix issue}

One could argue that these $\mathrm{RM} / R$-matrix comparisons are biased since they are based on the same RRP set. In a genuine evaluation work, the parameters of the analytical model are fitted on experimental data and then smaller $\mathrm{RM} / R$-matrix discrepancies are expected. Nevertheless the RM capture XS fitted will never be as good as a $R$-matrix curve because of the

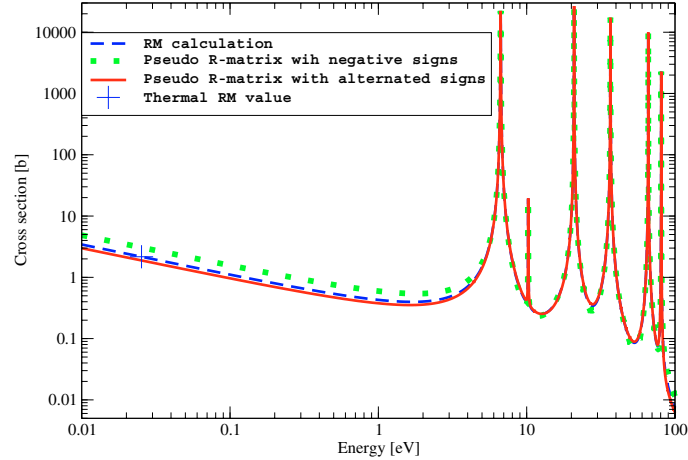

Fig. 2. Two pseudo $R$-matrix $\sigma_{\gamma}$ calculations of the ${ }^{238} \mathrm{U}$ with 23 individual radiative channels (assuming respectively all negative (dotted line) and alternated (solid curve) capture amplitude values) compared to the usual R-M calculation (long-dashed curve).

much smaller number of freedom degrees (the total capture width is only supplied) and of the more asymmetrical resonance shape introduced by the $R$-matrix analytical formula. The difference obtained between $R$-matrix and RM calculations on the thermal capture XS value $(-13 \%$ at least) might be stressful if the question of the negative levels would be unique. During a resonance evaluation work, the parameters of the lowest positive and negative resonances are fitted such as to adjust the calculation to the recommended thermal partial XS values. These parameter values rely on an ad hoc external level recipe chosen by the evaluator.

\section{External level (EL) representations}

$R$-matrix theory shows that the cross sections for scattering, capture, fission, etc. in a limited energy range depend not only on the "internal" levels but also on the "external" levels below and above. Problems arise in practical resonance fitting and parametrisation work because below the neutron threshold $(E<0)$ the compound levels are unobservable and therefore principally unknown. Since thermal XS values can be accurately measured, any RRR evaluation work must be normalised at thermal energy on the experimental data. Various approaches, more or less rigorous, have been developed to cope with the problem of unknown EL, depending essentially on the habit of the evaluator. The most worldwide spread methods [12] consist of:

A - simulating the unknown EL by equidistant "picket fence" or Monte Carlo sampled resonance ladders,

B - replicating the internal level sequence both below and above the internal region,

C - simulating EL by a smooth "background" XS added to the internal resonance contribution. This procedure was used for several nuclides $\left({ }^{237} \mathrm{U},{ }^{242} \mathrm{Cm}\right.$, etc. $)$ in JEF2.2,

D - describing the EL with a concise level-statistical formula rigorously derived from the reduced $R$-matrix analytical formulation which involves the neutron strength function and the distant-level parameter $R_{l}^{\infty}(E)$,

$\mathrm{D}^{\prime}$ - approximating the previous method by two broad resonances and a narrow bound level to ensure correct thermal cross sections. 
The non exhaustive list above shows that most of the RRR evaluations rely on approximated methods for the determination of the EL parameters essentially based on statistical trends. The spread of these techniques must warn direct evaluated file comparisons of the resonance parameters of the lowest energy resonances. In the future, RRR analyses should tend toward a unique convenient method and rigorous such as $\mathrm{D}^{\prime}$ although a part of unknown still remain in this latter via an undetermined scaling factor (i.e., $\Gamma_{\gamma}$ of the negative narrow level). Method $\mathrm{D}^{\prime}$ still requires some practical applications on fissile isotopes. Information on observed low lying levels, level density below $S_{n} g_{\gamma}$ and $g_{f}$ Westcott factors could be also incorporated in this method.

\section{Example of advanced calculations}

An area under development is the treatment of resolved resonance structures in other observables. An example of fluctuation reconstruction in the capture isomeric ratio $(\gamma I R)$ is given here below for the ${ }^{242} \mathrm{Am}$ compound nucleus $(\mathrm{CN})$. A first step for JEFF3.1 was already achieved with the evaluation of a smooth ${ }^{241} \mathrm{Am} \gamma \mathrm{IR}$ curve which covers the whole neutron reactor energy spectrum (0-20 MeV). In terms of fluctuating observables, the reader may also look over other works on ${ }^{235} \mathrm{U}$ fission data [13] and $v_{p}^{239} P u$ [14] for instance. The observation of fluctuating structures in other observables brings additional information for improving RRR XS fits.

\section{$5.1{ }^{241} \mathrm{Am} \gamma \mathrm{IR}$ gross energy dependency and errors}

The ${ }^{241}$ Am $\gamma$ IR versus energy (vertical-dashed line on fig. 3) has been recently evaluated for JEFF3.1 [1] by the way of a cubic error weighted polynomial fit on a large set of 17 integral and differential results. In addition a low energy asymptotic value $\left({ }^{241 \rightarrow 242 g} \gamma I R=0.91\right)$ for energies below $0.022 \mathrm{eV}$ and a high energy junction $\left({ }^{241 \rightarrow 242 g} \gamma I R=0.75\right)$ at $20 \mathrm{MeV}$, arbitrarily based on a TALYS [15] calculation with 10 discrete levels, were applied. It must be noticed that at high energy a considerable ambiguity remains since no experimental results exist above $300 \mathrm{keV}$ to confirm the model prediction.

A valuable achievement performed in parallel is the estimation of the associated standard deviations (SD) (see table 3) [1] stored in JEFF3.1 under file $40(\mathrm{MT}=102)$. The SD are essentially supplied by the ORIGIN software fit. At very low energy, the variance is set to the inverse of the sum of the inverse thermal data variances and above $300 \mathrm{keV}$ where no experimental data are available, the variance obtained from the polynomial fit is combined with half the difference between the fit connected at $20 \mathrm{MeV}$ to a ${ }^{241 \rightarrow 242 g} \gamma I R$ value equal to 0.75 and another possible fit based on a junction value of 0.5 .

Full variance and covariance evaluation is one of the major tasks of the next decade. In parallel to resonance data evaluation, uncertainties generation must be a priority. New methods to propagate uncertainties, as for instance on resonance parameters through the whole RRP evaluation procedure, are in development in codes like SAMMY [6] or CONRAD [16]. These methods, presented elsewhere in this conference, will not be debated here.

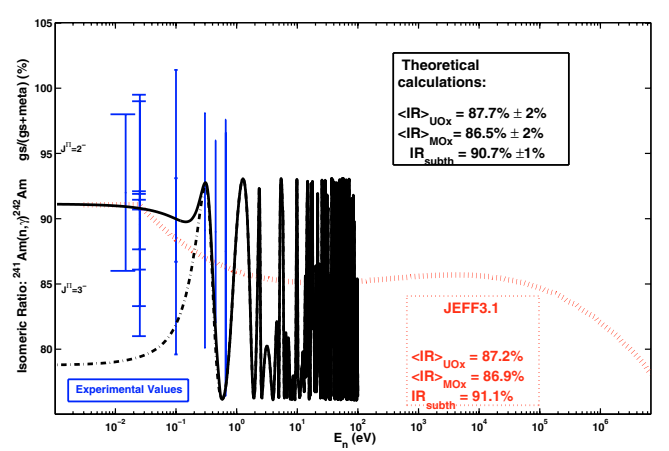

Fig. 3. Fluctuations superimposed to the smooth JEFF3.1 ${ }^{241 \rightarrow 242 g} \gamma I R$ evaluation (vertical-dashed curve). The thermal $\gamma I R$ tail can be induced by a $2^{-}$(solid curve) or a $3^{-}$(dotted-dashed line) bound level.

Table 3. Estimated relative standard deviations (SD) on ${ }^{241} \mathrm{Am} \gamma \mathrm{IR}$.

\begin{tabular}{|c|c|c|c|}
\hline \multicolumn{2}{|c|}{$\begin{array}{l}\text { Energy group boundaries } \\
{[\mathrm{eV}]}\end{array}$} & \multirow{2}{*}{$\begin{array}{c}\frac{\left.S{ }^{(241 \rightarrow 242 g} \gamma I R\right)}{2411242 g \gamma I R} \\
{[\%]} \\
0.69\end{array}$} & \multirow{2}{*}{$\begin{array}{c}\frac{S D(241 \rightarrow 242 m}{24 I R)} \\
{[\%]} \\
{[\%]}\end{array}$} \\
\hline 0.00001 & - $\quad 0.022$ & & \\
\hline 0.022 & $\begin{array}{ll}- & 0.1\end{array}$ & 0.42 & 3.54 \\
\hline 0.1 & $\begin{array}{l}-\quad 0.45 \\
\end{array}$ & 0.49 & 3.38 \\
\hline 0.45 & 0.8 & 0.58 & 3.88 \\
\hline 0.8 & - $\quad 1.6$ & 0.64 & 4.06 \\
\hline 1.6 & $-\quad 2.1$ & 0.68 & 4.56 \\
\hline 2.1 & 150. & 0.71 & 4.08 \\
\hline 150. & 40000 . & 0.54 & 3.15 \\
\hline 40000. & - $\quad 300000$. & 0.51 & 2.75 \\
\hline 300000 . & - $\quad 1000000$ & 1.75 & 8.47 \\
\hline 1000000 . & - $\quad 20000000$. & 11.36 & 38.77 \\
\hline
\end{tabular}

\subsection{Fluctuating $\gamma / R$ : a step beyond JEFF3.1}

Theoretical (preliminary) calculations [17], giant dipolar resonance model and $\gamma$-cascade stories based, were recently performed to investigate the possible fluctuating structure of the ${ }^{241} \mathrm{Am} \gamma \mathrm{IR}$. Owing to spin and parity selection rules between initial and final states, $\gamma$-transition probabilities were calculated for each allowed level transition belonging to the level scheme of the ${ }^{242} \mathrm{Am} \mathrm{CN}$. After derivation of the total $\gamma$-ray decay probability from an initial $J^{\pi}$ excited level given down to either the meta-stable $\left(J^{\pi}=5^{-}\right)$or the ground state $\left(J^{\pi}=1^{-}\right), \gamma I R$ were deduced. The ${ }^{241 \rightarrow 242 g} \gamma I R$ values calculated for an incident neutron energy slightly above $S_{n}$ (i.e., $\left.\mathrm{S}_{n}+1 \mathrm{eV}\right)$ were equal to $(93.1 \pm 0.8) \%$ and $(76.1 \pm 1.2) \%$ respectively for a $2^{-}$and $3^{-}$excited level. From the energies of the JEFF3.1 RRP set and a resonance spin assignment guess, the resulting $\gamma I R$ fluctuations in energy were simulated (see dotted-dashed or solid curves on fig. 3) by summing all calculated energy weighted thermal $\gamma I R$ resonance contributions; the energy weighting function being based on simple SLBW capture XS reconstruction.

By reference to the recent JEFF3.1 smooth evaluation of the ${ }^{241 \rightarrow 242 g} \gamma I R$ (vertical-dashed line on fig. 3) which was based on a comprehensive set of integral and differential results at thermal energy, one may guess with confidence the spin of the closest negative level which is likely $2^{-}$to match 
the fluctuating $\gamma I R$ simulation with the experimental overall trends.

\subsection{1 $\gamma I R$ improvement implications}

Practically in fuel assembly depletion and post irradiated experiment calculations, $\gamma I R$ are used on the form of capture branching ratios $(\gamma \mathrm{BR})$ weighted by the real capture reaction rate encountered in the case treated. A single $\gamma \mathrm{BR}$ average (A $\gamma \mathrm{BR})$ value, suited for a given spectrum type (i.e., $\mathrm{UO}_{2}, \mathrm{MOx}$ ), is supplied in the APOLLO2 (thermal cores) or in the ERANOS (fast cores) code systems. The corresponding JEFF3.1 A $\gamma$ BR values obtained from the smooth JEFF3.1 $\gamma I R$ curve are $87.2 \%$ and $86.9 \%$ respectively for $\mathrm{UO}_{2}$ and MOx spectra. The values derived from the fluctuating $\gamma I R$ theoretical curve $((87.7 \pm 2) \%$ and $(86.5 \pm 2) \%$ respectively), are in agreement with the JEFF3.1 adopted values. In order to better suit the core configuration treated, a procedure was recently added to the APOLLO2 code to calculate on line the relevant $\mathrm{A} \gamma \mathrm{BR}$ from the JEFF3.1 $\gamma I R$ point-wise data.

\section{The unresolved resonance range (URR) or the twilight zone}

Careful statistical analyses of the RRP obtained are essential to get valuable prior estimate of the average parameters (AP) to analyse unresolved resonance data. This statistical analysis stage is now made routinely using codes like ESTIMA [18] or SAMDIST (SAMMY's special module [6]) which byproducts are $s$-wave (or sometimes p-wave) strength functions $\left(S_{l}\right)$, average neutron reduced widths $\left(\Gamma_{n}^{l}\right)$, average radiative widths $\left(\Gamma_{\gamma l}\right)$, s-wave mean spacing $\left(D_{0}\right)$ and $R_{l}^{\infty}(E)$. Starting from prior average parameters, URR experimental data, after corrections of the experimental biases, can be fitted using Hauser-Feschbach theory (HF) and Moldauer prescription for overlapping resonances with codes like SAMMYFITACS [6] or recently CONRAD [16] to extract average parameters. Higher $l$-wave orders showing up with energy, an URR analysis brings obviously additional information on observables of higher l-wave degrees. High energy codes like TALYS [15] basically handle the same physics as low energy codes (SAMMY-FITACS, CONRAD, etc.) in this URR but the former evaluated average cross sections are derived from optical model (OM) parameters. The AP consistency between RRR analyses and OM calculations is for that matter studied in ref. [19]. It is clear that in a near future high energy codes will be able to extract AP for a right average XS shelf-shielding treatment in the UR domain. It is also manifest that an UR range will survive in the future to ensure the consistency between the RRR formalism and the OM calculations, and to treat specifically the mixing of large resolved resonances with small unresolved resonances. The resolution of this latter problem could be analog to the treatment of partially resolved class-II states mixed with unresolved class-I states to calculate the average fission XS of non fissile heavy nuclei (next section).

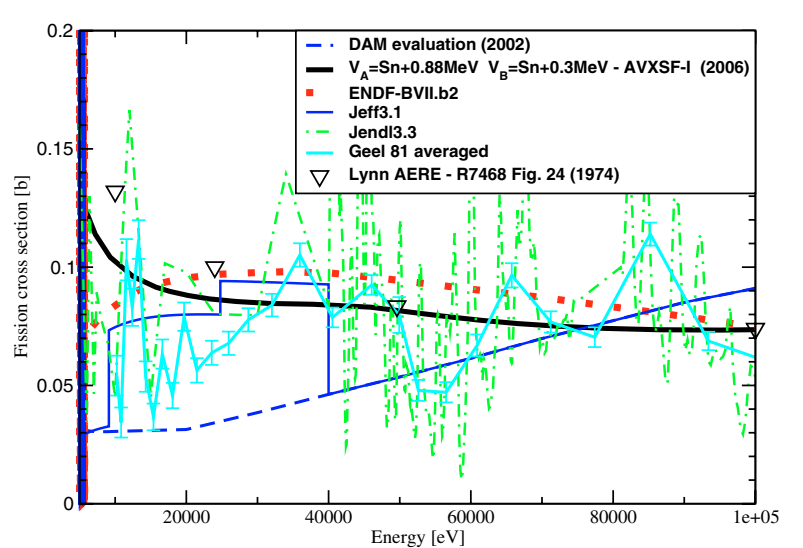

Fig. 4. Comparison of the major ${ }^{240} \mathrm{Pu}$ evaluated fission XS data (ENDF/B-VII. $\beta 2$, JEFF3.1 and JENDL3.3) with the experimental data (Geel 81) of Budtz-Jorgensen et al. [21], a recent AVXSF-I calculation (thicked curve) [21], a recent GNASH-TALYS calculation (dashed curve) [21] and the old Lynn calculation (downside triangles).

\section{The disused URR average fission cross section (AVFXS) of the non fissile actinides}

\subsection{Analytical average fission cross section formulae}

Because of their small neutronic impact in LWR reactor, non fissile nuclei AVFXS were frequently neglected in neutron resonance evaluation work. For instance, an overview on ${ }^{234} U$, ${ }^{238} \mathrm{U}$ and ${ }^{240} \mathrm{Pu}$ neutron evaluated fission cross sections in the UR region shows mainly simple smooth energy dependencies. Recently the gross energy fission structure of the XS has been calculated using TALYS-GNASH but one may say that the easiest way to locate the fission fluctuations at the right energy is simply to average broadly or acutely the experimental data.

A more careful examination of evaluated AVFXS shows that nearly no data files ${ }^{1}$ include statistical model with intermediate structure effect although some analytical subthreshold fission XS expressions by Lynn and Back [20] treat the fact that the fission strength is not distributed statistically over CN resonances. Such a formalism is implemented in the AVXSF code [20]. This program was tested in ref. [18] in the framework of the ${ }^{240} \mathrm{Pu}$ JEFF3.1 re-evaluation of the average XS and more recently [21]. Figure 4 shows the latest calculation (thicked curve) obtained with AVXSF-I under the approximation of "intermediate structure with class-II/class-I resonances moderately weak-coupled", with inner and outer formal barrier height values and discrete transition state energies close to those used by Lynn in his calculation (downside triangles on fig. 4).

Although the analytical model of Lynn and back is very efficient for giving the right magnitude of the average fission XS in the URR, this model does not reproduce the experimental fluctuations encountered in sub-threshold fission. This question can be solved with Monte Carlo methods.

\footnotetext{
1 The "intermediate structure with moderately weak coupling" form of Lynn and back was adopted by G. Vladuca et al. in some high energy evaluation works (see JEF/DOC-658 (1996) for instance).
} 


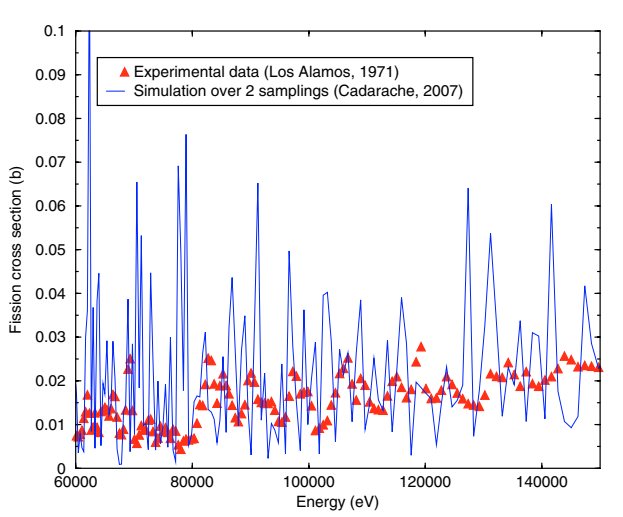

Fig. 5. Comparison of the ${ }^{242} \mathrm{Pu} \mathrm{MC}$ simulated fission cross section (solid curve) with the poor experimental data set of G.F. Auchampaugh et al. (1971-EXFOR No.10266-triangles).

\subsection{The Monte Carlo (MC) methods}

MC methods alternative to analytic expressions have been tried in the past such as ref. [22]. They are based on the sampling of ladders of both class-I and class-II states as well as their parameters, from the relevant distribution laws; then the coupling between each class-II and the neighbouring classI states can be treated on a case to case basis depending on the individual situation, and the XS be calculated from the resulting resonance parameters. The strength of the MC method is to fairly reproduce the amount of fluctuations observed in an experiment (see fig. 5). A new MC method being tried at Cadarache is willing to go further by fixing the energies of the resolved class-II quasi-particle states from the experiment rather than sampling them in order to locate the fission fluctuations at the right energy. In a second stage, the total fission width of the class-II quasi-particle states will be normalised on the experiment. These hybrid MC methods are possible only when at least a high resolution subthreshold fission measurement is available.

\section{Conclusions}

This paper has tried to answer to some practical questions met by a "resonance evaluator" but can not be exhaustive on a so wide topic. Fitting experiences have demonstrated that an experimental data base can not be comprehensive if differential and integrals results are not used together. The EL representation is somehow tricky and relies essentially on statistical quantities and measured thermal data. No absolute EL determination exists and developments are still needed in this area. The accuracy brought by a full $R$-matrix calculation is currently cancelled by the existing indetermination on the EL simulation. Nowadays the impact of resolved structures in other observables that XS (e.g., in IR, $v_{p}$ data, etc.) is under progress. Developments are also encountered in average subthreshold fission XS calculations. In particular MC methods are definitively a way to supply analytical methods when these latter are not able to reproduce observed fine fluctuations.

The presenting author would like to express his special thanks to the Cadarache Nuclear Data Group namely J.M. Ruggiéri, D. Bernard, C. de Saint Jean, E. Fort, B. Habert, O. Litaize, G. Noguère, E. Rich, C. Sage, O. Serot, J.C. Sublet, P. Talou and J. Tommasi for fruitful discussions in that matter. Enlightening discussions with A. Courcelle, A. Plompen and P. Ribon were appreciated. The author is also deeply indebted to N. Larson, J.E. Lynn and H. Weigmann for frequent support and invaluable help during the studies described.

\section{References}

1. O. Bouland, D. Bernard, Revised evaluation of the ${ }^{241}$ Am isotope, JEF/DOC-1086 (2004).

2. O. Bouland, Motivation for new ${ }^{241} \mathrm{Am}$ measurements, JEF/DOC-931 (2002); see also O. Bouland, Motivation for new measurements on ${ }^{241,242,243}$ Am isotopes, in Proc. of the Int. Conf. on Nucl. Data for Sc. and Tech., Santa Fe, 2004 (R.C. Haight et al., 2005), p. 211.

3. A. Koning et al., The JEFF-3.1 nuclear data library, JEFF Report 21, NEA/OECD (2006).

4. F.H. Fröhner et al., in Proc. of the Int. Conf. on Nucl. Data for Sc. and Tech., Antwerp, 1982 (K.H.Böckhoff, 1983), p. 211.

5. E.P. Wigner, L. Eisenbud, Phys. Rev. 72, 29 (1947).

6. N.M. Larson, Updated users' guide for SAMMY, Oak Ridge Report ORNL/TM-9179/R7 (2007).

7. G.M. Hale et al., Use of R-matrix theory in light element evaluations, in Proc. Int. Symp. Nucl. Data Eval. Methodology, Upton, New York, 1992 (World Scientific, 1993).

8. Z.P. Chen, At. Energy Sci. Technol., 29(4), 366 (1995).

9. M.C. Moxon, http://www.nea.fr/listsmh/ueval/msg00049.html.

10. F.H. Fröhner, Evaluation and analysis of nuclear resonance data, JEFF Report 18, NEA/OECD (2000).

11. O. Bouland, J. Bulg. Nucl. Soc., BgNS Trans. 10(2), 224 (2005).

12. F.H. Fröhner, O. Bouland, Nucl. Sci. Eng. 137, 70 (2001).

13. E. Fort, A. Courcelle, Fluctuations of fission data in the resonances with a channel-mode formalism (these proceedings).

14. D. Bernard et al., ${ }^{239} \mathrm{Pu}$ nuclear data improvements in thermal and epithermal neutron ranges (these proceedings).

15. A. Koning et al., TALYS-1.0 (these proceedings).

16. C. De Saint Jean et al., Proceedings of the PHYSOR-2006 Topical Meeting on Reactor Physics, Vancouver, 2006 (to be published).

17. D. Bernard, Theoretical calculations of ${ }^{241} A m(n, \gamma)^{242 g, m} A m I R$ via the CN process, Proc. of WONDER-2006, Cadarache, 2006.

18. O. Bouland, Re-evaluation of the ${ }^{240} \mathrm{Pu}$ cross sections in the URR, JEF/DOC-917 (2002).

19. E. Rich et al., Generalisation of the SPRT method: application to the ${ }^{242} \mathrm{Pu}$ cross sections in the URR (these proceedings).

20. J.E. Lynn, Systematics for neutron reactions of the actinide nuclei, AERE Harwell Report No. 7468, 1974 (unpublished).

21. H. Weigmann and O. Bouland, Exp. and eval. sub-threshold fission cross sections, CEA/LEPh Tech. Note, 2007 (unpublished).

22. H. Weigmann et al., Nucl. Phys. A 438, 333 (1985). 